\title{
Experimental substantiation of the effectiveness of the bioactive layer of the titanium dental implant
}

\author{
Khabilov NL, Usmanov F.K. Rashidov RA \\ Tashkent s State Dental Institute Tashkent, Uzbekistan.
}

\section{Email address:}

khabilov@mail.ru (Khabilov NL)

To cite this article:

Gulnoza Ruzieva, Komila Porsokhonova, Bekhzod Abdullaev. Epilepsy and affective disorders: depression as a suicide risk (review article). Journal of research in health science. Vol. 1, No. 2, 2018, pp. 31-37. DOI 10.26739/2523-1243

\section{doi http://dx.doi.org/10.26739/2523-1243/-2018-1-2-5}

\begin{abstract}
In the experiments it was shown that the preliminary deposition of chitosan on the bioactive surfaces of helical titanium implants improves the morphological characteristics of osseointegration after implantation in the femoral bone of the crudes.

Revealed additional osteoconductive effect of tricalcium phosphate and chitosan + layer, resulting in around implants is more intense and advancing at timing neoplasm of bone in combination with the morphological characteristics and express remodeling seal surrounding bone.
\end{abstract}

Key words: titanium implants, chitosan, bone tissue, remodeling, experiment.

Relevance. To assess the toxicity and monitor the processes of survival of the implants under study from the point of view of biochemistry, we studied the dynamics of such biochemical indices of the blood of experimental animals as the activity of aminotransferase enzymes and protein fractions of blood (toxicity markers), as well as macroelements calcium, magnesium, phosphorus exchange, violation osteogenesis and development of osteoporosis [ 6 ].
The most important structural components of bone tissue are osteotropic macro- and microelements. For the human body and animals, the importance of calcium, phosphorus, magnesium and other elements is very great.

Calcium and phosphorus are part of the basic structural component of bone oxypatite. Excretion of these macronutrients from the body leads to the loss of not only the mechanical properties of bone tissue, but also disrupts the 
Khabilov NL, Usmanov F.K. Rashidov RA. Experimental substantiation of the effectiveness of the bioactive layer of the titanium dental implant.

balance of mineral metabolism in the body, thereby causing a chain of pathological changes in all systems and organs $[1,5]$.

It is known that the formation of osteoporosis is accompanied by a decrease in the level of magnesium in bone tissue, which contributes to the instability of hydroxyapatite crystals and negatively affects energy metabolism [ 8 ].

In connection with the importance of mineral metabolism in osteogenesis, a number of investigators studied the dynamics of changes in the amount of calcium, magnesium and phosphorus in blood serum and bone when studying the survival of implants with calcium phosphate and other sputterings [ 2, 6, 8, 10 ].

Objective: To assess the effectiveness of the application of the domestic bioactive layer of tricalcium phosphate / chitosan on dental implants according to the morphological state of bone tissue in the experiment.

Objects of research - titanium dental implants with bioactive tricalcium phosphate / chitosan coating and bone rabbits after implantation.

Materials and methods of research. In the work 18 thoroughbred rabbits with a body weight of 4200-4300 were used. The animals were kept in the vivarium of the Intercollegiate Scientific Research Laboratory (TINA) on a standard diet, taking into account the provisions of the international convention on "Rules for working with experimental animals" ( European Communities Council Directives of 24 November 1986, $86 \backslash$ $609 \backslash$ EEC). Under general anesthesia animals were implanted in the hind pawInvestigated implants. As a comparison, another group of animals was implanted with titanium implants without sputtering.

Titanium dental implants with a spray of bioactive tricalcium phosphate / chitosan coating and titanium dental implants without the deposition of a bioactive layer were established after general anesthesia. Animals injected intravenously into the ear vein with a freshly prepared solution of sodium ethaminal at a dose of $40 \mathrm{mg} / \mathrm{kg}$, then after the onset of deep sleep, a $0.5 \%$ solution of novocaine was administered topically subcutaneously. After removing the wool at the site of the future operation and aseptic treatment of the skin with an alcohol solution of iodine, skin sections of 4-5 $\mathrm{cm}$ with a subsequent cut of muscle layers were made. By flaking out the skinmuscle flap, access was obtained to the head of the femur. Osteoplastic surgery for the implantation of a dental implant is made by a drill with a diameter of 1 $\mathrm{mm}$ with a speed of $1000 \mathrm{rpm}$. with irrigation with physiological solution. Then, a serial drilling was carried out with a drill with a diameter of 1.5 with a rotation speed of $800 \mathrm{rpm}$. with irrigation with physiological solution. Rotating movement of a special key set implants, after their installation. When applying the seams, a standard layer technique (4-0 vitryl for inner layers, 4-0 nylon for the skin) was used.

The general state of laboratory animals was monitored hourly during the first day, and once a day during the next 90 days of the experiment. Before the study and throughout the experiment, clinical signs of possible intoxication were recorded: general condition of animals, feed and water intake, change in body weight (every three days), characteristics of behavior, intensity and nature of motor 
activity, coordination of movements, response to external stimuli, frequency and depth of respiratory movements, the state of the wool and skin, mucous membrane color, tail position, quantity and type of fecal masses.

For the entire period of the experiments, all laboratory animals were kept in standard vivarium conditions and were on a high-grade laboratory food diet with free access to water.

In the dynamics (after 1,2,3 months after implant placement), the X-ray of the implantation site was performed.

\section{Histomorphological studies.}

For conducting morphological studies after euthanizing animals made a fence fragment proximal department great e tibia bones. Material fixed at $10 \%$ solution neutralformalin, a after commit decalcified at $25 \%$ solution organic acids Trilon B. After traditional postings and fill at paraffin produced serial sections thickness $8 \mathrm{~m}$. The preparations were stained with hematoxylin and eosin. Microscopic examination was carried out using a light microscope MICMED-2 from increase at 40,100, 200 and 400 time.

\section{RESULTS AND DISCUSSION \\ Results of histo- morphological studies of bone tissue of rabbits.}

After 1, 2 and 3 months after the operation, femoral removal was carried out followed by a resection of the bone sampling head for histological examination from the sites, where the graft adjoined. Material fixed at $10 \%$ solution neutral formalin, a after commit decalcified at $25 \%$ solution organic acids Trilon B. After traditional postings and fill atparaffin produced serial sections thick $8 \mathrm{~m}$. Preparations stained hematoxylin and eosin.

When morphological examination of bone tissue during implantation of the dental implant with the deposition of the bioactive layer after 1 month in contrast to the control group and 2 groups (implants without sputtering) In the bone tissue of the rabbits of group 3 , a significant $40-50 \%$ filling of the implant surface outside a loose, sometimes dense connective tissue. Fibroblasts with collagen fibers leaving them are visible, as well as elements of revascularization .

AT preparations of rabbits of group 3 after 2 months in The regenerated zone reveals a formed lamellar bone tissue surrounded by sections of the soft-fibrous connective tissue with single capillaries. By periphery of the areas of the osteoplastic material, active osteoblasts are determined, which is due to the formation of the newly formed bone tissue (in Figure 1 it is indicated by an arrow). AT Active macrophages are found in the regeneration zone and osteoclasts that perform resorption of the implant. The osteoplastic material is gradually replaced by a connective tissue and forming bone beams. AT interosseous cells are determined elements of myeloid- lipoid bone marrow (Figure 1).

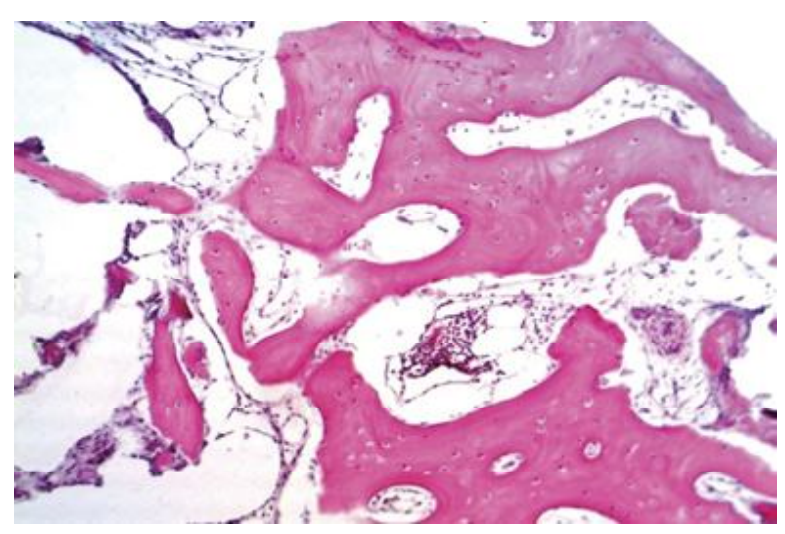

Fig.1. Formation of bone tissue after application of osteoplastic material 2 months after the implantation of dental implants with the deposition of a bioactive layer. Coloring GE. Ув: о к.10 x об.1 0. 
Khabilov NL, Usmanov F.K. Rashidov RA. Experimental substantiation of the effectiveness of the bioactive layer of the titanium dental implant.

Also, in the histological study of biopsy specimens in The bone girders of dense spongy bone were found in the regeneration zone. AT interosseous cells was determined by the soft-fibrous connective tissue with a large number of newly formed capillaries (in Figure 2 indicated by arrows). By edge of bone beams single osteoclasts, also there are single osteoblasts.

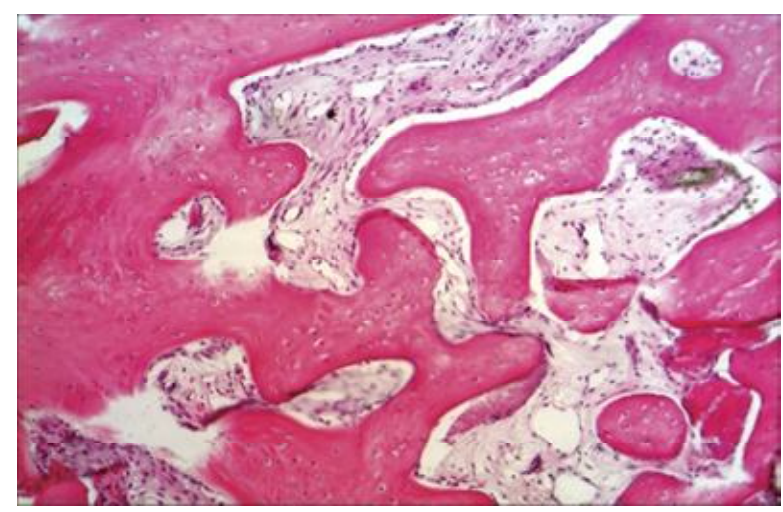

Figure 2. Formation of bone tissue after the application of osteoplasticmaterial 2 months after the implantation of dental implants with the deposition of a bioactive layer. Coloring GE . У : 0 к.10 х об.1 0.

In the course of the experiment, in order to exclude the deformation of the surrounding bone tissue after the implant application, a piece was taken from a bone tissue that was distant by $1 \mathrm{~cm}$. The study did not reveal atypical or dysplastic changes in the structure of bone tissue. In addition, the formation of bone beads of the lamellar bone, represented by a small number of osteocytes and dense compact bone substance (indicated by the arrows in Figure 3). Between the bony beams there are channels with loose unshaped connective tissue containing fibroblasts and fibrocytes, there are single vessels of the microcirculatory bed.

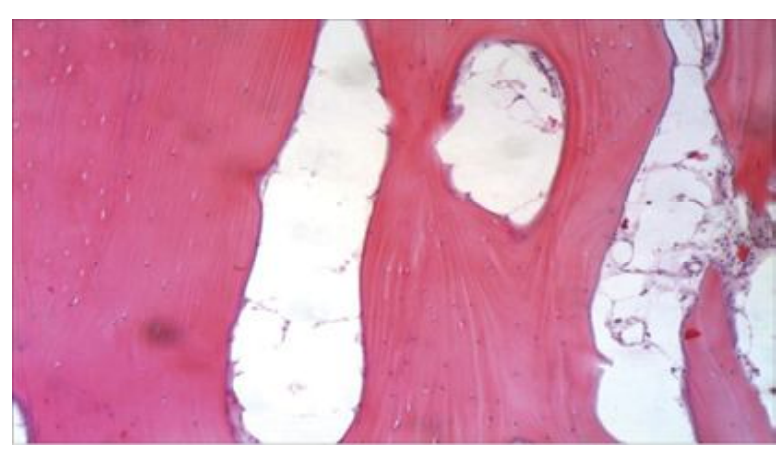

Figure 3. Mature bone tissue, distant from the implantation zone after 3 months of experiments. Staining with hematoxylin and eosin. Coloring GE. Ув: ок.10 х об.10 .

Results of X-ray studies of bone tissue of rabbits after implantation.

Comparison X-ray images and results of morphological studies allowed to establish the development of contact osteogenesis (formation of bone tissue on the surface of the implant), starting from 30 days of research in animals of the 3rd group. In dental implantation with the deposition of the bioactive layer under study migration of osteogenic cells directly on the surface of the implant with the participation of fibrin fibers of the blood clot was noted. The presence in the blood clot of the progngators (cells for bone creation) promoted the acceleration of the regeneration process (Fig. 4). A comparative analysis of the periods of osteogenesis in animals of the 2nd and 3rd groups made it possible to state that the dental implant with bioactive sputtering possesses physical properties closest to natural tissues. The surface of bioactive sputtering tricalcium phosphate+ chitosan provided adsorption of proteins and adhesion of cells, organic and mineral components of bone matrix.

Titanium implants with tricalcium phosphate + chitosan coating on comparison of from titanium implants without sputtering more actively partici pate 
at organotypic restructuringzone bone defect. AT experienced groups tracked change rate of osteogenesis and quality of bone reshaping. AT particular, It was it is established, what in rabbits in 3 groups the first manifestations organotypic restructuring were observed already on the 60th day with the formation of massive bone beams and hematopoietic foci, whereas in the animals of the 2nd group manifestations were less are expressed and emerged only on 90th day. In addition, the number of newly formed bone tissues and intensity of organotypicrestructuring prevails in the group with use of tricalcium phosphate + chitosan spraying. This trend manifested itself as on early, So and on remote periods of experiments (Figures 5, 6, 8).

$\mathrm{X}$-ray data and morphological research demonstrated the difference at quality bone restructuring in rabbits of 2 and 3 groups. Bioactive sputtering on a dental implant tricalcium phosphate + chitosan showed a pronounced Ability to organotypic reorganization as early as the 30 th day. On the contrary, titanium implant without a bioactive coating showed the least osteogenic activity at all times of observation.

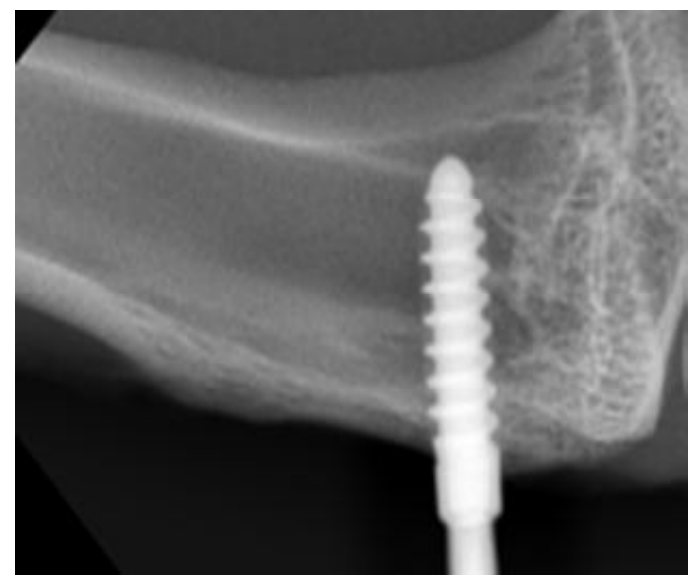

Fig. 4 . Bone tissue around the implant with a deposition on the 7 th day . Around the implant is a rarefaction of bone structures. Fig. 5. Bone tissue around the implant with a deposition on day 21 .
Noted filling the implant surface the outside is loose connective tissue.

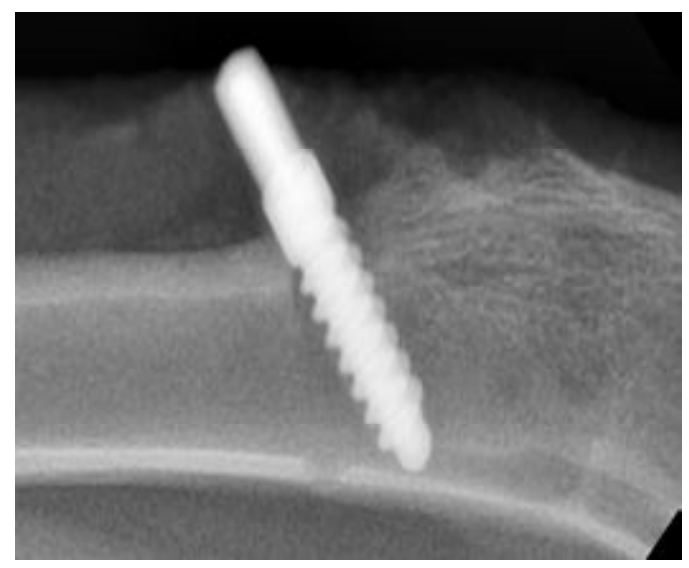

Fig. 5 . Bone tissue around the implant with a deposition on day 21 . Noted filling the implant surface the outside is loose connective tissue.

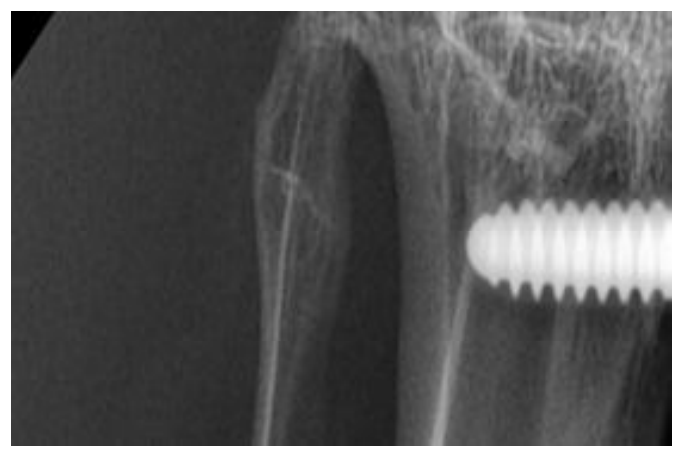

Fig. 6. Bone tissue around the implant with a deposition after 1 month. Noted filling the implant surface externally loose, sometimes dense connective tissue Fig. 7. Bone tissue around the implant with a deposition after 2 months. Strong integration ofimplant with deposition in the bone.

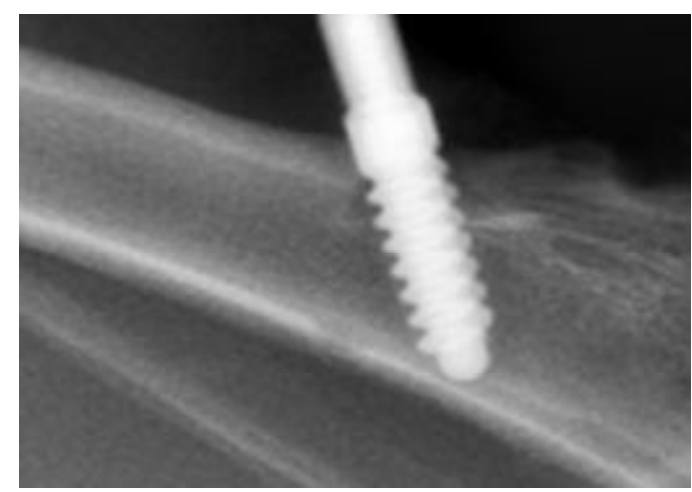

Fig. 7. Bone tissue around the implant with a deposition after 2 months. Strong integration ofimplant with deposition in the bone. 
Khabilov NL, Usmanov F.K. Rashidov RA. Experimental substantiation of the effectiveness of the bioactive layer of the titanium dental implant.

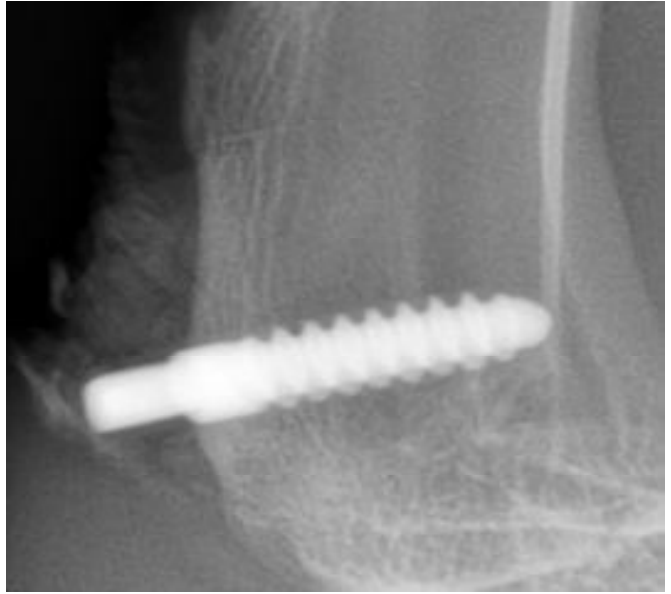

Fig. 8 . Bone tissue around the implant with a deposition after 90 days. A complete healing of the bone tissue around the titanium implant with sputtering was noted.

\section{Conclusions}

$\mathrm{H}$ and the results of their own research found that the composites of chitosan and tricalcium phosphate have no negative impact on the health of the experimental animals.

Morphological analysis of the bone tissue of the head of the femur allowed to study the processes of bone formation after implantation of dental titanium implants with the deposition of bioactive layer tricalcium phosphate + chitosan and evaluate the effect on process of reparative regeneration of the bioactive layer. It is obvious that processes of bone regeneration tricalcium phosphate + chitosan deposition has a positive effect.
Histological studies and control X-rays confirm the intensive osteogenesis when installing titanium implants with the deposition of tricalcium phosphate and chitazone .

The analysis of histological preparations showed that the domestic composite does not break the process of bone formation and dental implants are reliably and stably integrated at the site of implantation. Reliable osseointegration depends on the material in the bioactive sputtering and the design features of the implant itself.

$\mathrm{X}$-ray images and results of morphological studies confirm the signs of osseointegration : Implant permanence (ankylosis), intimate contact with bone tissue without signs of inflammation, absence of signs of thinning of bone tissue or the interval between the implant and the bone on X-ray, all adjacent tissues in a normal state. Bioactive compositetricalcium phosphate and hitazona is osteoinductive, serves as a framework, in which regenerates bone tissue. Resorption of the bioactive layer occurs gradually, the restoration of bone tissue occurs by type of complete healing. Research results allow us to draw a conclusion about safety for public health and the effectiveness of bioactive layer for dental implants chitosan + trialcium phosphate. 


\section{References/Литература}

1. Vasilenko Yu.K. Biological chemistry. - Moscow : Higher School. 1978. - 381 p.

2. Gizatullin R.M. The use of nano-gel of calcium hydroxyapatite and porous nickel titanium for the treatment of destructive forms of chronic periodontitis // Kazan Medical Journal. - 2009. Volume 90 .- ? 3 . - S. - 438-440 .

3. Zaitseva VM, Liflyandsky VG, Marinkin V.I. Applied medical statistics / Textbook .- St. Petersburg - Publishing house "Foliant" .- 2003.- 432 p.

4. Makarov V.G. et al . Directory. Physiological, biochemical and biometric indicators of the norm of experimental animals. СПБ .: Publishing house "LEMA" .- 2013. - 116 with.

5. Nazarenko GI, Kishkun AA . Clinical evaluation of laboratory results. - Moscow: Medicine .- 2000. - 540 p.

6. Pavlova LA, Pavlova TV, Nesterov AV Morphological analysis of the bone defect using a titanium implant treated with a sandblast method with various composite coatings in the dynamics of the first month of regeneration // Scientific statements of BelSU. - 2010. - No. 4 (75). - Vol. 9. - P. 58-63 .

7. The provisions of the international convention on "Rules for the work with experimental animals" ( European Communities Council Directives of 24 November 1986, 86 \609 \EEC).

8. Trifanova EB, Ganzha AA, Gyulnazarova SV, Burmatova A.Yu. Features of markers of mineral metabolism when implanting the spokes into an osteoporotically rearranged bone tissue // Fundamental research. 2014 . - № 6 (part 7) . - P. 1428-1431 .

9. Pavlova PV, Pavlova LA, Bokova EN, Pavlov IA, Nemiikin ON, Nesterov AV // European Jornal of Natural History -2010. - 4.-P. 4-7]).

10. Burger EL, Patel V. Calcium phosphate as bone graft extenders.// Ortho pedics. - 2007. - 30. - P. 939. 